\title{
BEAR, une station de mesure pour l'océan Arctique
}

\author{
R. Loisil(1), L. Eymard( ${ }^{(2)}$, N. Amarouche ${ }^{(1)}$, J.-M. Panel(3), A. Lourenço(2), \\ A. Matulka ${ }^{(4)}$, A. Weill(4), F. Vivier ${ }^{(2)}$, M. Dechambre ${ }^{(4)}$, A. Viola ${ }^{(5)}$, \\ V. Vitale ${ }^{(5)}$, S. Argentini ${ }^{(5)}$ et H. Kupfer ${ }^{(6)}$ \\ (1) Division technique de l'Institut national des sciences de l'Univers (DT INSU), \\ France \\ (2) Laboratoire d'océanographie et du climat : expérimentation \\ et approches numériques (LOCEAN), France \\ (3) Centre d'études de la neige, Grenoble (CENG) - CNRM/GAME, \\ Météo-France/CNRS, France \\ (4) Laboratoire atmosphères, milieux, observations spatiales (LATMOS), France \\ (5) ISAC, Istituto di Scienze dell'Atmosfera e del Clima (CNR), Italie \\ (6) AWI, Alfred-Wegener Institut für Polar und Meeresforschung, TU-Dresden, \\ Allemagne
}

\section{Résumé}

Dans le cadre du projet ANR OPTIMISM, une station météorologique a été mise au point afin d'accéder au bilan d'énergie dans l'océan Arctique via le couplage de cette station avec le système ICE-T de détermination de l'épaisseur de la glace (Ice Thickness determination). Les premiers essais technologiques et scientifiques de cette station nommée BEAR (Bilan d'énergie arctique et recherche) sont décrits. Des résultats préliminaires, obtenus lors d'une campagne expérimentale en avril-mai 2010 à $\mathrm{Ny}$ Ålesund (Spitzberg) au-dessus d'une surface de neige/glace, sont notamment présentés et mis en perspective.

\section{Abstract}

\section{BEAR: a measurement station} for the Arctic Ocean

A meteorological mast was developed in the framework of the OPTIMISM ANR project in order to get the energy budget in the Arctic Ocean when coupled with the ICE-T (Ice Thickness determination) system. First technological and scientific trials of this meteorological mast named the BEAR system (Budget of Energy for Arctic Research) are described. Preliminary results from an experiment at $\mathrm{Ny}$-Ålesund (Spitsbergen) in April-May 2010 over an ice/snow surface are more particularly presented and put into perspective.
$P$ our les prochaines décennies, les modèles climatiques prévoient des élévations de température dont les plus importantes sont localisées dans les zones arctiques. Une évolution est déjà observable dans ces régions. Elle se manifeste par la fonte des glaces de mer dont les conséquences seront significatives en termes de formation des eaux profondes et de bilan radiatif du globe. Les régions arctiques apparaissent donc comme une composante essentielle de la vulnérabilité du système climatique global.

Ce constat justifie des observations accrues et une analyse approfondie des mécanismes physiques à l'interface océan-atmosphère afin de mieux comprendre l'ensemble des processus gouvernant l'évolution de la masse de glace et d'en améliorer la prise en compte et la représentation dans les modèles climatiques.

Dans le cadre du projet OPTIMISM (Observing dynamical and thermodynamical Processes involved in The sea Ice Mass balance from In Situ Measurements) de l'Agence nationale de la recherche (ANR) [Vivier, 2010], nous avons proposé comme défi et développement technologique innovant un prototype de flotteur autonome, destiné à obtenir des mesures in situ de l'épaisseur de glace, des paramètres radiatifs et des flux gouvernant l'équilibre du bilan de masse dans les régions arctiques.
Ce flotteur doit être déployé dans plusieurs régions arctiques : une polynie côtière (étendue d'eau libre au cœur de la banquise) et des zones marginales de glace (zones frontières entre la banquise et l'eau libre). Le système de mesure est constitué de deux modules reliés entre eux : un module pour les mesures océaniques (système ICE-T) et un module pour les mesures atmosphériques (système BEAR).

Le module pour les mesures océaniques est le système ICE-T (Ice Thickness) décrit dans Vivier (2010). Il est constitué d'une bouée équipée qui a déjà montré son adéquation à des mesures dans la glace et son opérationnalité potentielle au Spitzberg. Les mesures à bord de cette bouée sont dévolues à la couche de surface océanique : elles permettront, d'une part, l'étude du rôle du mélange et de la formation de la glace « frazil » sous la banquise et, d'autre part, l'étude des ondes de gravité de surface.

Le module de mesures météorologiques BEAR, dont il est question dans cet article, surmonte la bouée ICE-T et utilise des capteurs météorologiques et radiatifs ayant déjà fait leurs preuves dans les régions arctiques.

Ces capteurs sont censés effectuer des mesures au même niveau dans la couche de surface atmosphérique (couche à flux constant) :

- une mesure de vent (en module et direction) ; 
- une mesure de température et humidité de l'air ;

- une mesure complète du rayonnement incident et réfléchi dans les domaines visible et infrarouge.

Ce système est conçu pour pouvoir estimer les flux turbulents de chaleur sensible, de chaleur latente et de quantité de mouvement par des méthodes simplifiées, très largement utilisées au-dessus de la mer (les méthodes « bulk»), ainsi que le bilan radiatif de surface. Couplée à ICE-T dans le concept de la bouée du projet OPTIMISM, cette station météorologique doit pouvoir fonctionner de façon autonome pendant plus d'une année dans l'ambiance de l'océan Arctique, notamment dans la région du Spitzberg, en se comportant comme une bouée dérivante dans les zones d'eau libre et comme une bouée fixe par rapport à la glace lorsqu'elle se fige dans la glace.

Dans une première partie, nous décrirons la conception technique du système $\mathrm{BEAR}$ et nous présenterons les premiers éléments d'une campagne de mesure technologique hivernale en altitude au col du Lac Blanc, à l'Alpe d'Huez, de décembre 2009 à janvier 2010. Nous expliciterons ensuite les méthodes envisagées pour estimer les flux turbulents en nous inspirant, en particulier, des résultats d'expériences internationales en régions arctiques. Nous définirons ainsi notre stratégie et justifierons notre choix en termes de méthodes bulk.

Enfin, dans les troisième et quatrième parties, nous illustrerons, à partir de résultats choisis, le fonctionnement $\mathrm{du}$ système au Spitzberg, à Ny-Ålesund, en avril-mai 2010, en termes de mesures obtenues et de bilan d'énergie associé. À titre de comparaison, nous présenterons également des résultats issus de deux stations de mesure, l'une italienne, l'autre allemande, situées dans la même zone.

Nous pourrons alors établir de premières conclusions et nous envisagerons les perspectives d'utilisation du système BEAR couplé avec ICE-T dans le cadre des stations automatiques autonomes OPTIMISM.

\section{Le système BEAR}

\section{Description du système}

Le système BEAR a été conçu pour un fonctionnement optimal en régions arctiques et il est le fruit d'une analyse de l'instrumentation existante. Il comprend une mesure de vent, une mesure de la température de l'air et de l'humidité relative et une mesure des composantes montantes et descendantes du rayonnement dans les domaines visible et infrarouge. Il ne constitue donc pas à lui seul une station météorologique complète dans la mesure où des paramètres de servitude tels que la pression atmosphérique, la température de la surface de neige ou de glace ainsi que la hauteur de glace plus neige seront estimés dans le futur par le module ICE-T.

Les mesures de vent (module et direction) sont réalisées à partir du système Young 05103-45. Cet instrument, qui succède au «Young » classique déjà utilisé en Arctique et en montagne ${ }^{(1)}$, a été développé pour être robuste, résister à des températures fortement négatives et être peu sensible au givre.

Pour les mesures de température et d'humidité relative de l'air, le système HMP155 de Vaisala a été choisi, associé avec un abri ventilé naturellement. Ce système est employé de façon préférentielle dans les instrumentations en régions froides.

Concernant les mesures du rayonnement visible et infrarouge, le CNR4, nouveau système de Kipp et Zonen, de faible poids $(900 \mathrm{~g})$, a été adopté en raison de ses spécificités arctiques déjà démontrées par son prédécesseur, le CNR1. De plus, les possibilités offertes en termes de chauffage et de ventilation du capteur complet avec un minimum de consommation d'énergie devraient assurer une sécurité pour les

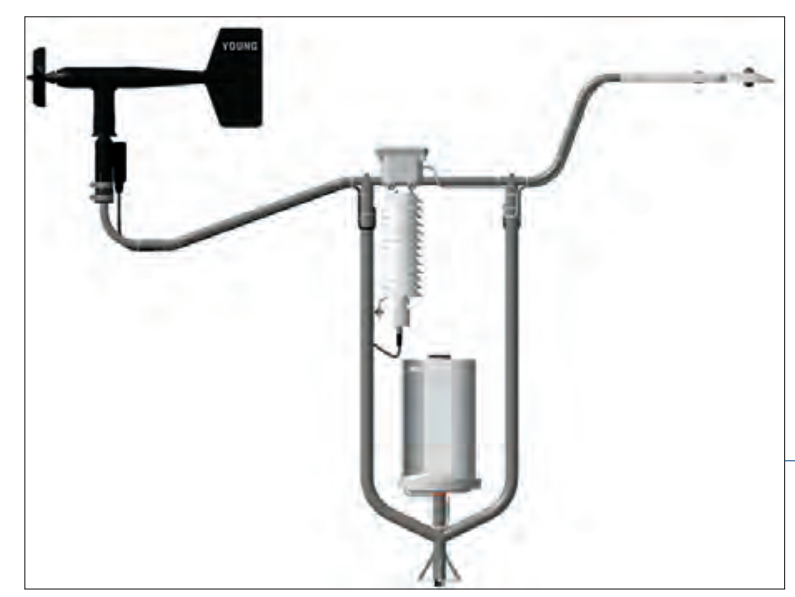

mesures infrarouges, élément important durant l'hiver polaire. Pour le pyranomètre, mesurant le rayonnement visible incident et réfléchi, la gamme spectrale se situe entre 300 et 2800 nanomètres tandis que pour le pyrgéomètre (mesurant le rayonnement infrarouge incident et émis par la surface) la gamme spectrale s'étend de 4500 à 42000 nanomètres.

Bien que le système ait été prévu pour fonctionner de façon autonome à partir de batteries, il nous a paru intéressant de vérifier si une petite éolienne permettrait au moins de charger une batterie destinée au seul chauffage des capteurs infrarouges. L'éolienne retenue est une FORGEN 500 dont le seuil de démarrage est voisin de quelques mètres par seconde et qui a fait l'objet d'une étude en Antarctique (rapport UNAVCO, 2008).

L'ensemble des éléments de la station a été installé sur un mât météorologique en aluminium d'une masse voisine de $2 \mathrm{~kg}$ et d'une hauteur d'un mètre. La masse de l'ensemble est inférieure à $9 \mathrm{~kg}$.

Sur la figure 1, on peut voir une esquisse du système montrant l'ensemble des capteurs presque au même niveau, avec l'éolienne à axe vertical au bas de la figure. Une antenne Iridium pour communication satellitaire est prévue sur l'axe horizontal métallique situé au-dessus de l'éolienne (support des capteurs).

Pour les premiers tests d'évaluation du système, il convient de noter que l'instrumentation est alimentée par le réseau électrique, ce qui permet, dans un premier temps, d'effectuer un bilan de consommation, de quantifier les batteries qui seront nécessaires pour un fonctionnement autonome sur une année et d'optimiser les cadences d'échantillonnage pour l'acquisition des données. Les batteries de l'ensemble du système OPTIMISM - dont le module BEAR - seront par la suite stockées dans le corps de la bouée ICE-T.

L'équilibre du système OPTIMISM (ICE-T et BEAR) n'est pas discuté dans cet article mais le système BEAR

Figure 1 - Schéma conceptue du système BEAR (Division technique de l'Institut national des sciences de l'Univers, DT INSU). 
a toutefois été réalisé de façon à pénaliser le moins possible l'équilibre de l'ensemble : des études de stabilité statique ont été effectuées et des études de stabilité dynamique sont en cours.

\section{La campagne technologique au col du Lac Blanc}

Le système BEAR a été préparé pour fonctionner à côté d'une station de mesure à 2800 mètres d'altitude, au col du Lac Blanc à l'Alpe d'Huez, avec pour objectif principal d'analyser les conditions probables de givrage et de diagnostiquer la formation du givre. Le givrage est en effet une question cruciale au vu des conditions atmosphériques susceptibles d'être rencontrées dans le cadre du projet OPTIMISM. Nous n'analysons ici que le fonctionnement du système BEAR.

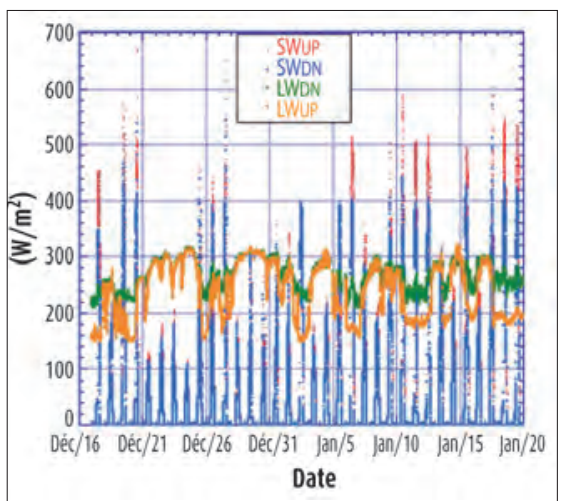

Figure 2 - Rayonnement visible incident $\left(S W_{D N}\right)$ et réfléchi $\left(S W_{u p}\right)$ et rayonnement infrarouge incident $\left(\mathrm{LW}_{\mathrm{DN}}\right)$ et émis par la surface ( $\left(\mathrm{WW}_{\mathrm{UP}}\right)$ lors des essais au col du Lac Blanc, à l'Alpe d'Huez.

Les mesures de température et d'humidité n'ont pas présenté de caractéristiques particulières si ce n'est une humidité relative par rapport à la glace souvent supérieure à $100 \%$, ce qui, avec des températures très négatives, a probablement dû conduire à du givrage. Concernant le rayonnement, nous représentons sur la figure 2 les rayonnements visibles (SW) et infrarouges (LW) montant (UP) et descendant (DN). Nous remarquons des valeurs du maximum du rayonnement visible incident $\left(\mathrm{SW}_{\mathrm{DN}}\right.$, en bleu) dépassant $450 \mathrm{~W} / \mathrm{m}^{2}$, ce qui paraît très fort à cette période. Cette augmentation artificielle, attribuable à la composante diffuse du rayonnement visible incident, peut être une conséquence d'effets topographiques. Les rayonnements infrarouge incident $\left(\mathrm{LW}_{\mathrm{DN}}\right)$ et émis par la surface $\left(L W_{U P}\right)$ ont un ordre de grandeur réaliste mais il faut être circonspect compte tenu d'un givrage important observé les 23 et
29 décembre et peut-être permanent entre ces journées, non décelable sur les mesures de BEAR.

Toutefois, rien dans le comportement du vent, que ce soit le module ou la direction, ne permet a priori de diagnostiquer un effet de givrage et nous n'avons donc pas trouvé de critère simple de prévision de ce phénomène à des fins de chauffage préventif des capteurs de rayonnement. La photo 1a montre l'anémomètre couvert de givre, semblant s'orienter correctement. L'hélice tourne mais sans doute le fonctionnement est un peu altéré, ce que nous ne retrouvons pas dans les mesures. En effet, nous ne distinguons pas de dysfonctionnement apparent sur le signal mesuré pour la vitesse du vent, comme on peut le voir sur la figure 3 où nous avons visualisé en rouge les périodes de saturation par rapport à la glace avec une forte probabilité de givrage. Il en est de même pour les mesures radiatives : les capteurs du CNR4, mesurant la composante réfléchie, apparaissent partiellement obstrués par le givre (photo $1 \mathrm{~b}$ ) et ont donc un fonctionnement dégradé certain mais qui reste difficilement quantifiable.

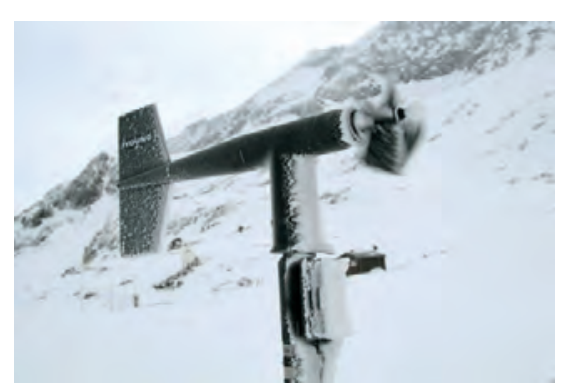

Photo 1a - L'anémomètre Young le 23 décembre 2009.

Figure 3 - Vitesse de vent en fonction du temps. En rouge, sont mis en évidence des épisodes d'humidité saturée par rapport à la glace.

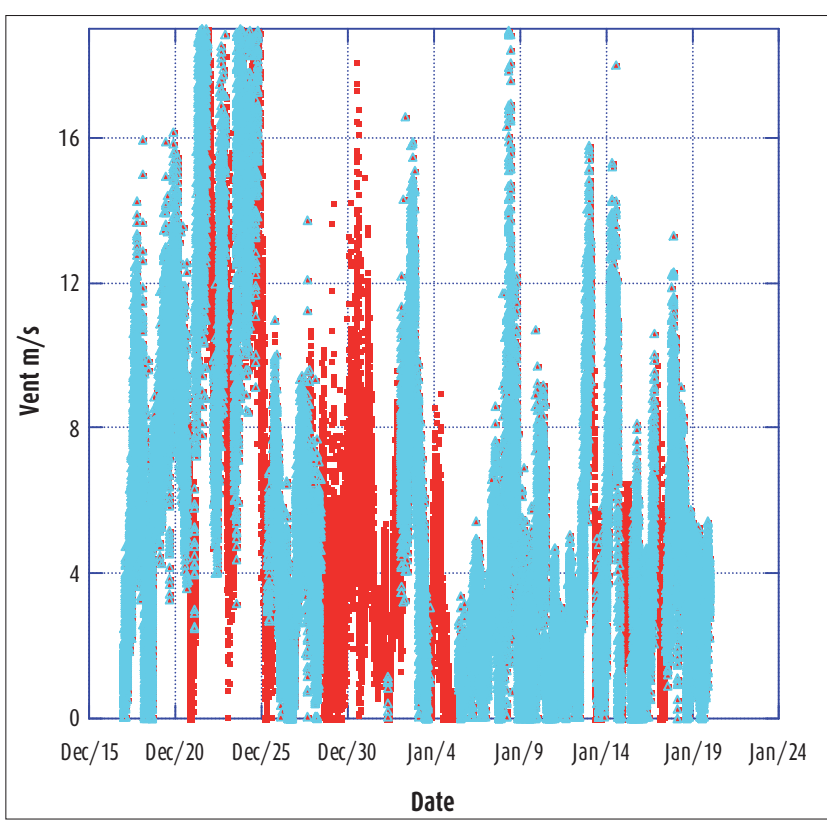

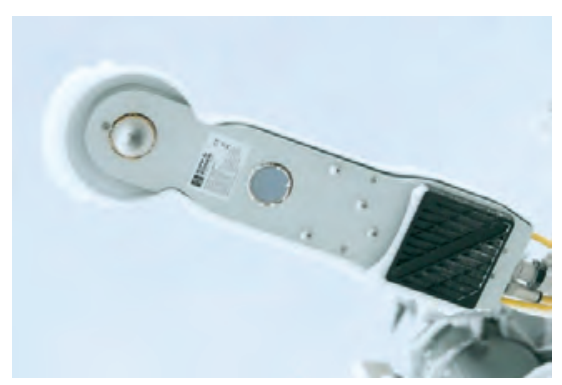

Photo 1b - Le CNR4 (composante réfléchie), instrument permettant les mesures du rayonnement visible et infrarouge.

Dans les conditions atmosphériques opératoires du projet OPTIMISM, il faudra donc, si l'on veut opérer précautionneusement, d'une part, définir un chauffage conditionnel du capteur radiatif en cas de probabilité de givrage (afin d'économiser les batteries de chauffage) et, d'autre part, bien distinguer les périodes où les données risquent d'être potentiellement incorrectes compte tenu de la saturation par rapport à la glace.

\section{Bilan d'énergie en surface et méthodes bulk}

\section{Le bilan d'énergie en surface}

Si nous écrivons le bilan d'énergie de surface à la surface de la glace, de la neige ou de l'eau en contact avec l'air, nous obtenons :

$S W_{D N}-S W_{U P}+L W_{D N}-L W_{U P}=H+E+T_{r}(1)$

$S W_{D N}$ et $S W_{U P}$ sont les rayonnements visibles incident et réfléchi : composantes mesurées par le pyranomètre. $L W_{D N}$ et $L W_{U P}$ sont les rayonnements infrarouge incident et émis par la surface : composantes mesurées par le pyrgéomètre. $H$ et $E$ représentent respectivement les flux turbulents de chaleur sensible et de chaleur latente. L'ensemble de ces composantes est exprimé en $\mathrm{W} / \mathrm{m}^{2}$. 
$T_{r}$ représente la partie de l'énergie transmise par l'atmosphère à la surface ou transmise par la surface à l'atmosphère, avec la convention de signe suivante : $T_{r}$ est positif si le flux est transmis par la surface à l'atmosphère. $T_{r}$ inclut les flux conductifs dans la glace et la chaleur latente de fusion de la glace. $T_{r}$ doit être estimé par le système ICE-T à partir de mesures de température à différentes profondeurs. $T_{r}$ peut également être estimé à partir du bilan radiatif net, $R_{n}$, directement mesuré par le bilanmètre CNR4 du système BEAR.

$R_{n}=S W_{D N}-S W_{U P}+L W_{D N}-L W_{U P}$

Une analyse des différences entre ces deux estimations de $T_{r}$ sera particulièrement utile pour affiner le bilan d'énergie.

\section{Les flux bulk dans la couche de surface à flux constant}

Pour estimer les flux turbulents, on se propose d'utiliser une méthode simple d'estimation appelée méthode " bulk » (de couche), très largement utilisée. Cette méthode consiste à exprimer les flux turbulents, qui sont des paramètres d'ordre 2 (covariances entre les variables), en fonction du gradient vertical (entre la surface et une hauteur $h$ ) des paramètres moyens tels que le vent, la température et l'humidité spécifique (qui sont d'ordre 1). Les méthodes bulk reposent sur une première hypothèse selon laquelle les paramètres moyens au niveau $h$ sont représentatifs d'un domaine d'altitude appelé couche à flux constant ou couche limite de surface (généralement les 10 premiers mètres de l'atmosphère), dans lequel les flux turbulents atmosphériques sont constants (à $10 \%$ près). Les méthodes bulk s'appuient sur le concept de similitude de Monin-Obukhov (voir par exemple Businger et al., 1971) qui implique que l'ensemble des profils verticaux moyens du vent horizontal, de la température et de l'humidité dans la couche limite de surface sont proches de profils logarithmiques, avec des écarts (par rapport à de véritables profils logarithmiques) qui sont fonctions de la stabilité atmosphérique.

Les formulations bulk vont alors consister à relier les flux turbulents de surface aux paramètres moyens mesurés à deux niveaux : un niveau $h$ (mesuré par rapport à la surface) et un niveau $\mathrm{z}_{0}$, où $\mathrm{z}_{0}$ est la longueur de rugosité, caractéristique des effets de surface et pour laquelle le profil logarithmique du vent horizontal moyen extrapolé vers la surface s'annule. Les flux turbulents s'expriment alors sous la forme :

$\tau=\rho \cdot C_{D} \cdot U^{2}$

$H=\rho \cdot c_{p} \cdot C_{H \cdot} \cdot\left(\theta_{s}-\theta\right) . U$

$E=\rho \cdot L_{v} \cdot C_{E} \cdot\left(q_{s}-q\right) \cdot U$

avec $\rho$ la masse volumique, $U$ le vent horizontal moyen, $\theta$ la température potentielle moyenne et $q$ l'humidité spécifique moyenne. Tous ces paramètres sont mesurés au niveau $h$.

$\theta_{s}=T_{s}$ est la température moyenne estimée au niveau $\mathrm{z}_{0 \mathrm{~T}}$, et $q_{s}$ est l'humidité spécifique moyenne estimée au niveau $\mathrm{z}_{0 \mathrm{q}}$, où $\mathrm{z}_{0 \mathrm{~T}}$ et $\mathrm{z}_{0 \mathrm{q}}$ sont des longueurs de rugosité spécifiques pour la température et l'humidité (voir Andreas, 2002).

$\tau=\rho . u_{*}^{2}$ est le flux de quantité de mouvement.

$H$ et $E$ sont les flux de chaleur sensible et latente déjà énoncés dans la section précédente.

$u_{*}$ est la vitesse de frottement.

$c_{p}$ est la capacité calorifique de l'air à pression constante.

$L_{v}$ est la chaleur latente de vaporisation de l'eau.

Les coefficients $C_{D}, C_{H}$, et $C_{E}$ sont respectivement les coefficients de traînée aérodynamique, thermique et hydrique, paramétrés en fonction des flux (ou de la stabilité) et très différents selon les auteurs.

Le problème posé par les méthodes bulk est le suivant : pour chacune des variables météorologiques moyennées (vent horizontal, température et humidité), on dispose de mesures à un niveau d'altitude $h$ (variable dans le temps) par rapport à la surface (de neige, de glace ou d'eau). En revanche, la notion de température de surface $T_{s}$ est ambiguë car le mot « surface » peut se révéler avoir plusieurs significations selon les types de mesures effectuées (thermodynamiques ou radiométriques) et le niveau d' « altitude » considéré (c'est-àdire la valeur de longueur de rugosité de surface utilisée). Lorsque la formulation avec la température radiative de surface est utilisée (par inversion des mesures radiatives infrarouges) plutôt que la mesure de la température thermodynamique de la surface (par sonde), la précision de cette température de surface est de l'ordre de $0,5{ }^{\circ} \mathrm{C}$ en moyenne (Perovich et al., 2002 ; Persson et al., 2002).

\section{Méthodologie et paramétrisations bulk}

La précision de l'estimation des flux turbulents est davantage liée aux paramétrisations utilisées qu'aux incertitudes sur les paramètres moyens mesurés, de telle sorte que, si les paramérisations étaient universelles, les méthodes indirectes bulk seraient bien plus précises que des mesures directes de flux turbulents (Weill, 1991). La méthodologie que nous projetons de mettre en œuvre consiste à calculer les flux $H$ et $E$ en utilisant, en premier lieu, les paramétrisations « classiques » du type de celles utilisées par Fairall et al. (2003), Grachev et al. (2007), et Grachev et Fairall (1997). Dans les cas stables où la température de l'air au niveau $h$ est supérieure à la température de la surface, les paramétrisations de Beljaars et Holtslag (1991), Andreas (2002), Grachev et al. (2007), et De Bruin et al. (2000) pourront être considérées comme des paramétrisations de référence.

Dans les régions arctiques, la température de la surface (glace ou neige) est le plus souvent inférieure à la température des premiers mètres de l'atmosphère. Nous sommes donc concernés par des couches dites stables ou stablement stratifiées, impliquant des paramétrisations spécifiques applicables à ce type de stratification. Depuis peu, nous disposons d'informations sur le comportement de la turbulence des couches de surface en régions arctiques, en particulier grâce aux expériences SHEBA de 1998 au Groenland (Persson et al., 2002 ; Grachev et al., 2007). D'autre part, il existe une littérature abondante et largement usitée sur les couches stables que l'on trouve en hiver ou la nuit dans nos régions (voir par exemple Beljaars et Holtslag, 1991). Pour encadrer les estimations des flux en conditions stables, nous nous proposons donc de mettre en œuvre les paramétrisations les plus utilisées disponibles dans la littérature.

Une méthode d'estimation des flux bulk proposée par Price et Dunne (1976) mérite notre attention. Cette méthode s'adresse à des couches de surface de faible épaisseur et a été appliquée et validée au-dessus de la neige fondante par Arck et Sherer (2002). Les travaux de Lüers et Bareiss (2010), lors de l'expérience ARCTEX 2006 au Svalbard, ont justifié son application lors des périodes de transitions au printemps mais aussi pendant l'hiver arctique où les couches de surface atmosphériques risquent d'être ténues. Nous utiliserons donc cette méthode pour les estimations de flux 
turbulents présentées dans la section «Évaluation des flux de chaleur sensible et latente bulk - Incertitudes associées »».

Une étape ultérieure consistera à analyser la vraisemblance des estimations en balayant les plages d'incertitude relevant de plusieurs paramétrisations réputées significatives ou utilisées dans des modèles (CCSM, ECMWF, ARCSyM et GFS) qui, d'après Brunke et al. (2006), corroborent les mesures d'Andreas (2002). Notons toutefois que la démarche expérimentale de la mesure « locale » diffère de la démarche de la modélisation météorologique ou climatique qui représente, en général, des effets moyens à d'autres échelles.

\section{La campagne expérimentale au Spitzberg}

\section{Implantation du système BEAR}

La campagne expérimentale s'est déroulée sur le site de Ny-Ålesund au Spitzberg, du 27 avril au 10 mai 2010, sur une zone enneigée, à proximité d'une tour météorologique de 30 mètres édifiée par les équipes italiennes d'ISAC-CNR. Afin de ne pas être sous son influence, le système BEAR a été installé à environ 70-80 mètres de celleci, ce qui permet de limiter les effets de pente, d'ombrage et de sillage. Les coordonnées GPS de BEAR sont $78^{\circ} 55^{\prime} 48^{\prime \prime}$ Nord et $11^{\circ} 52^{\prime} 12^{\prime \prime}$ Est.

La figure 4 présente un schéma d'implantation et la carte correspondante du site expérimental. Sur le schéma d'implantation (figure $4 a$ ), on peut se rendre compte que l'orientation de l'axe CNR4-Young (mesures du rayonnement et du vent) a été optimisée par rapport aux effets d'ombrage possibles des différents reliefs (correspond à l'axe SudNord) et que le site peut être considéré comme plat. La photo $2 \mathrm{a}$ (panoramique à 180 degrés) illustre cette considération. Sur la photo $2 \mathrm{~b}$, nous avons choisi de représenter une vue vers l'ouest de la station BEAR. Comme la position du soleil n'est à l'ouest que vers $17 \mathrm{~h}$ UTC, alors que l'élévation solaire est proche de 15 degrés, la station BEAR ne devrait pas être affectée.

La hauteur de neige est estimée à partir d'un réglet fixé sur le support cylindrique du mât du système BEAR. Par

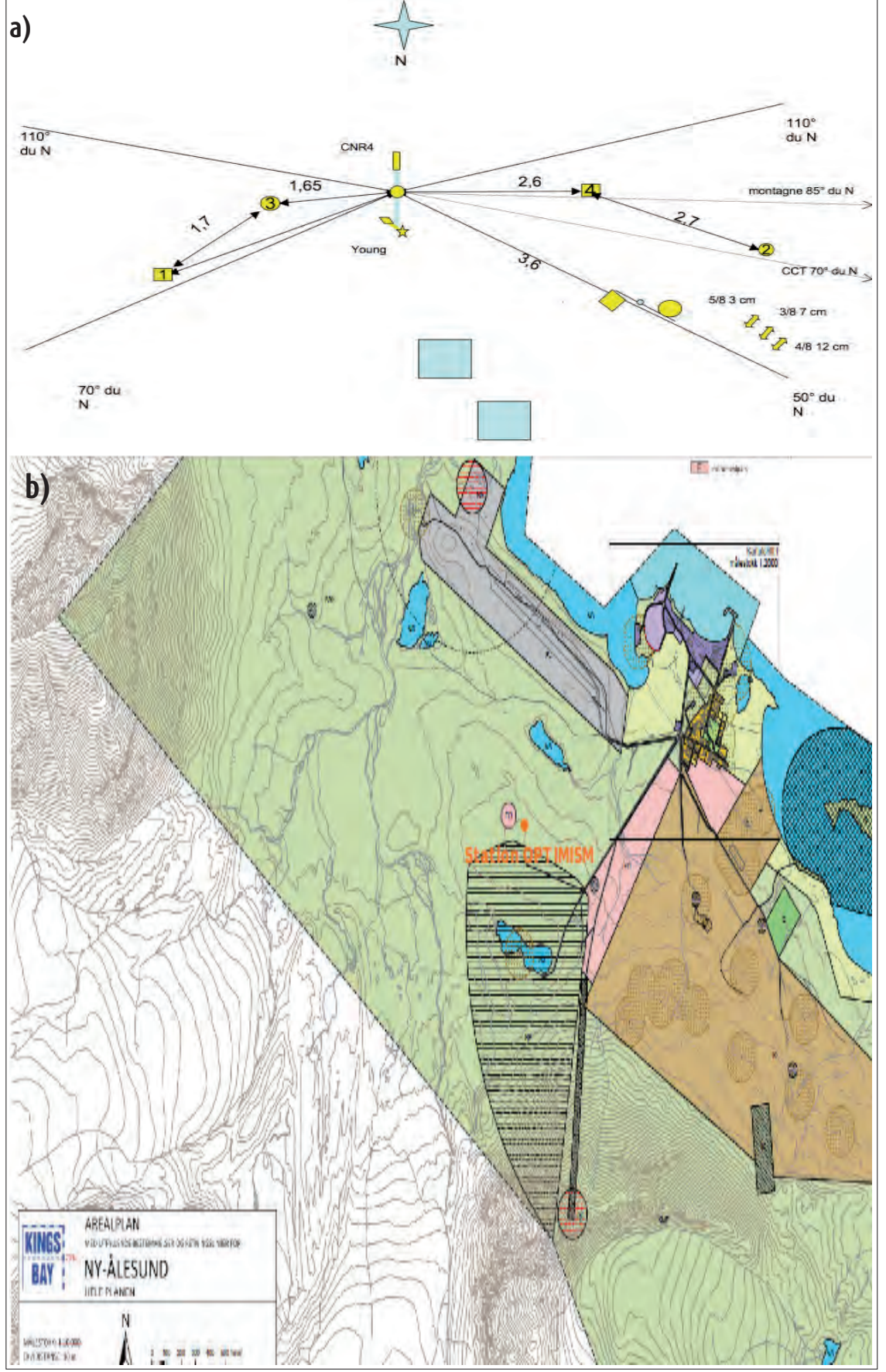

AFigure 4 - Schéma d'implantation (a) et localisation géographique du site (b).

Photo $2 b$ -
Vue vers l'ouest de la station BEAR

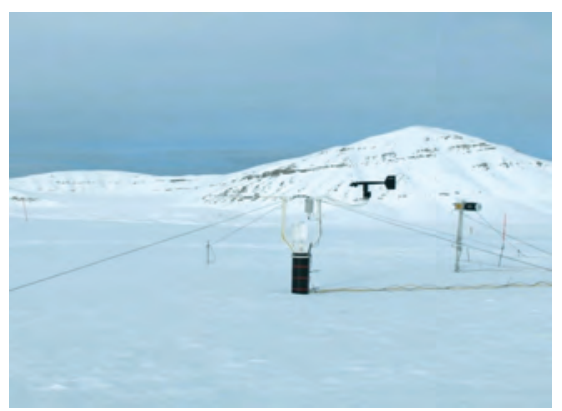

montrant la tour météorologique italienne CCT

ainsi que l'emplacement du mât BEAR (OPTIMISM).

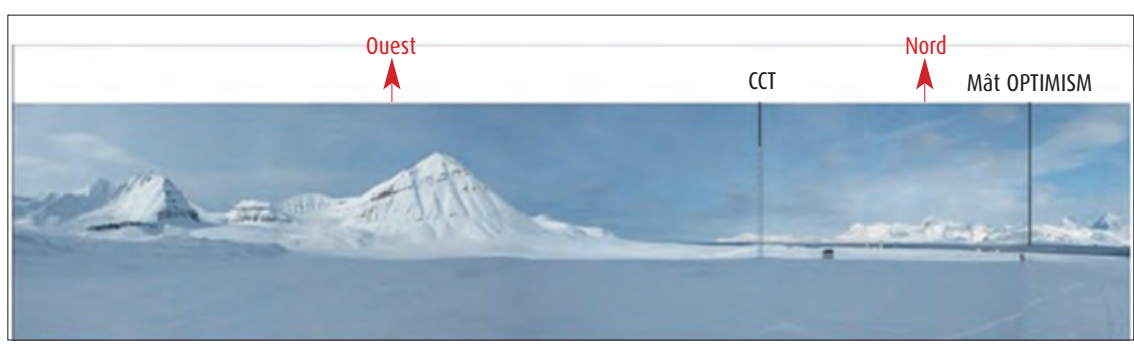


ailleurs, nous disposons, près de la station BEAR, d'un dispositif photographique automatique permettant, d'une part, de visualiser des effets de givrage sur la station et, d'autre part, de surveiller la surface de neige à raison d'une photographie toutes les 15 minutes. Enfin, le dispositif expérimental inclut quatre mesures de température de surface de la neige (à $0,5 \mathrm{~cm}$ sous la surface de la neige) ainsi que trois mesures effectuées respectivement à $3 \mathrm{~cm}, 7 \mathrm{~cm}$ et $12 \mathrm{~cm}$ de la surface. Il est fait en sorte qu'en cas de neige, à partir d'un système de deux disques et de deux grilles, les mesures dites de surface soient ramenées à la même profondeur sous la couche de neige que celle-ci se soit formée ou ait été érodée. Mises en œuvre par le CENG, ces mesures sont indiquées par les chiffres $1,2,3$ et 4 sur le schéma d'implantation (figure 4a).

\section{Comparaisons des mesures}

Dans cette première étude, nous nous intéressons aux comparaisons entre les mesures effectuées par le système BEAR et celles issues de la tour italienne (mesures de vent, température et humidité à $2 \mathrm{~m}$ au-dessus du sol et mesures de rayonnement visible et infrarouge effectuées au sommet du mât à $32 \mathrm{~m}$ ). Nous bénéficions également des mesures météorologiques (vent, température, humidité et rayonnement) de la station franco-allemande AWIPEV, située beaucoup plus au sud-est, proche des bâtiments et également plus proche de la côte. Cette station est exposée à des conditions météorologiques locales différentes de celles du site où se trouvent le système BEAR et la tour italienne. Dans la suite de cet article, nous noterons FR les données concernant BEAR, IT celles de la tour italienne, et $\mathrm{G}$ celles de la station AWIPEV des équipes allemandes.
Les mesures du système BEAR (FR) se sont révélées être situées à $1,7 \mathrm{~m}$ de la surface de la neige et ce malgré des apports neigeux qui ont rapidement été érodés certains jours (par fusion ou transport par le vent). Dans les autres stations (tour italienne et station francoallemande AWIPEV), les mesures ont été réalisées, en théorie, au niveau $2 \mathrm{~m}$ mais, dans les faits, à $1,5 \mathrm{~m}$ du plan zéro à cause de la neige tombée pendant l'expérience. Seules les mesures italiennes de rayonnement sont effectuées en altitude (au sommet du pylône, à $32 \mathrm{~m}$ ). Cette différence de niveau de mesure ainsi que la non-réponse parfaite en cosinus des capteurs radiatifs impliquent un rapport entre les surfaces concernées par les mesures proche de 300 (rapport d'homothétie des surfaces en relation avec les hauteurs d'observation) pour les composantes réfléchie/ émise par la surface, sans compter des différences, aussi bien sur les composantes descendantes que montantes, compte tenu de l'atténuation du rayonnement sur 32 mètres.

\section{Vent, température et humidité}

À présent, nous allons nous intéresser aux paramètres dynamiques et thermodynamiques que nous comparerons à partir de régressions linéaires entre les mesures FR et les mesures IT et G. De façon conventionnelle, les mesures de ces variables sont généralement ramenées à $10 \mathrm{~m}$. Nous ne choisissons pas ici cette normalisation, car les couches de surface dans les régions arctiques sont supposées d'épaisseurs nettement inférieures.

La figure 5 fournit une comparaison des mesures des modules de vitesses du vent, des températures et des humidités.

Figure 5 - Diagramme de dispersion des mesures de la tour italienne (IT) et de la station AWIPEV (G) en fonction de BEAR (FR) pour :

a) le module du vent ; b) la température de l'air ; c) l'humidité relative.

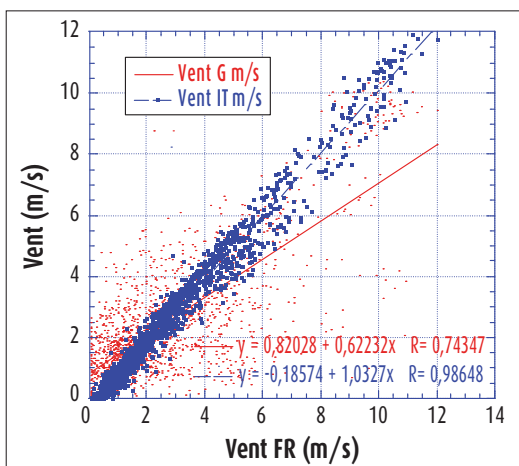

(a)

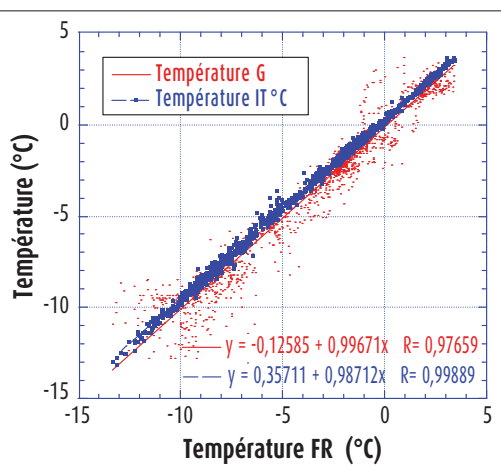

(b)
Sur les régressions linéaires (figure 5a), nous observons pour le vent des mesures FR et IT très corrélées avec une pente voisine de 1 mais une dispersion non négligeable. En revanche, les intensités du vent $\mathrm{G}$ ont un comportement statistique différent qui peut être lié à des propriétés locales (effets de bâtiment, topographie, etc.).

On retrouve cette différence de comportement si on analyse le vecteur vent entre les sites FR, IT et G pendant la campagne (figure 6). Des noyaux de direction des vents sont observables sur les trois enregistrements. Toutefois ces modes sont moins intenses sur le site G.

Entre les sites FR et IT, un biais de rotation voisin de $15^{\circ}$ est sans doute dû à une erreur d'orientation des mesures du vent et n'affecte que peu le vent horizontal. Comme on vient de l'évoquer à partir des régressions sur le vent horizontal moyen, les atténuations du vent sur le site $\mathrm{G}$ correspondent aux effets locaux dans une zone topographique différente, entourée de bâtiments, ce qui devrait avoir des impacts sur l'état de la surface de la neige et, en conséquence, sur le rayonnement de la surface.

Examinons maintenant les caractéristiques de la température de l'air et de l'humidité relative (figures $5 \mathrm{~b}$ et $5 \mathrm{c}$ ). En ce qui concerne la température de l'air, les comportements des sites FR et IT sont proches, avec un biais de $0,36{ }^{\circ} \mathrm{C}$. Par ailleurs, le comportement de la station FR reste proche de celui de la station $\mathrm{G}$, malgré une dispersion plus importante des mesures issues de la station AWIPEV. Pour l'humidité relative, le biais entre les mesures FR et IT est faible $(5,8 \%)$, le niveau de corrélation fort et la pente de la régression voisine de 1. Ce n'est pas le cas de la corrélation entre FR et $\mathrm{G}$ qui montre un biais de $19 \%$, caractéristique de conditions locales différentes. Le module du vent y est en moyenne plus faible, ce qui va jouer un rôle sur les paramètres thermodynamiques et hydriques.

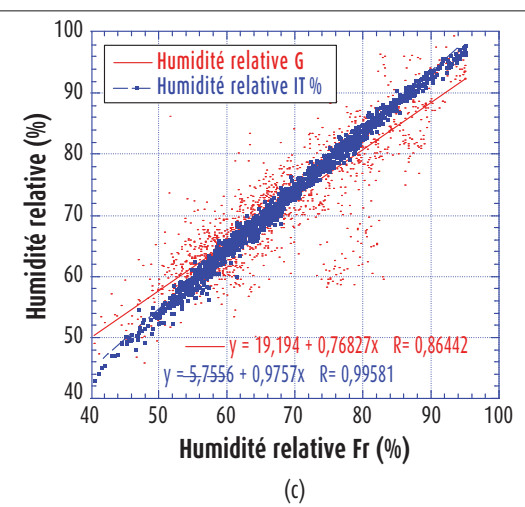




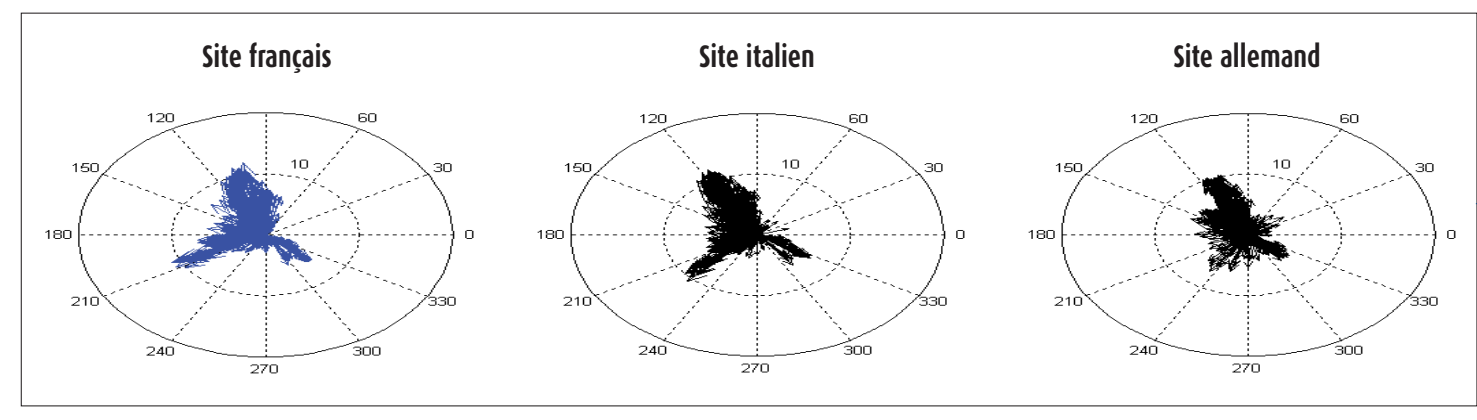

Figure 6 -

Comparaison

entre vecteurs vent

sur les sites FR,

IT et $\mathrm{G}$, pendant

la campagne.

automatiquement toutes les 15 minutes, près de la station BEAR.

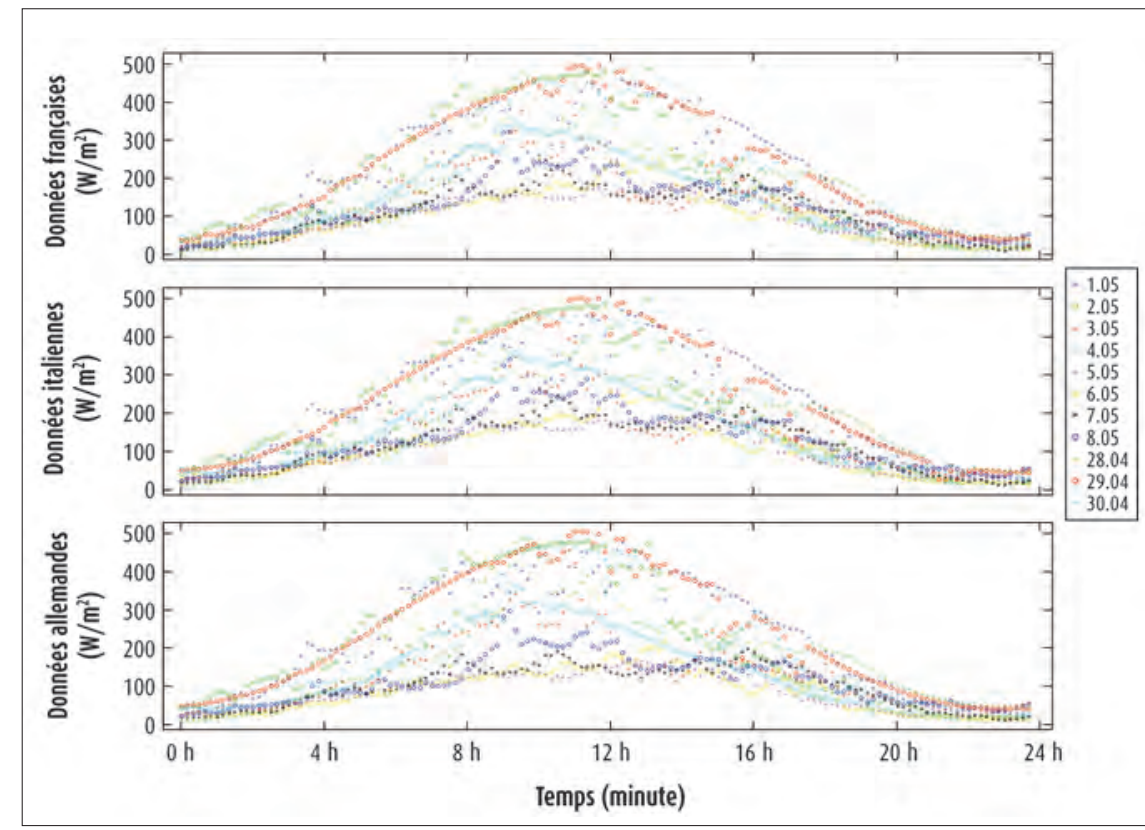

Figure 7 - Rayonnement visible incident $\left(\mathrm{SW}_{\mathrm{DN}}\right)$ mesuré sur les trois sites (FR, IT et G).
Examinons maintenant les comparaisons des composantes incidentes (DN) et réfléchie/émise par la surface (UP) du rayonnement visible (SW) et infrarouge (LW) entre les trois sites (figure 8).

Le rayonnement visible incident (fig. 8a) présente une cohérence importante entre les trois sites avec notamment une dispersion très faible entre les stations FR et IT. Les trois sites ont un rayonnement visible incident peu différent puisqu'il est indépendant de la surface, exception faite de la composante diffuse.

Sur la régression tracée pour le rayonnement visible réfléchi (fig. 8b), on retrouve la proximité des mesures FR et IT (meilleure corrélation, pente voisine de 1) avec un biais de $13 \mathrm{~W} / \mathrm{m}^{2}$ à l'origine. Pour $\mathrm{G}$, la corrélation avec FR est légèrement plus faible. On
Pour l'ensemble des trois variables considérées, on constate que les mesures FR et IT sont très proches (corrélations fortes, pente voisine de 1 et biais à l'origine faible), ce que nous espérions et qui valide la mesure du vent, de la température et de l'humidité fournies par la station BEAR pendant la période d'observation.

\section{Rayonnement visible et infrarouge}

En premier lieu, nous allons examiner chacune des quatre composantes du rayonnement (rayonnement visible incident et réfléchi, rayonnement infrarouge incident et émis par la surface) pour l'ensemble des stations. Nous aborderons ensuite les estimations de l'albédo ainsi que celles du bilan radiatif net.

La figure 7 illustre essentiellement les cycles diurnes observés sur l'ensemble des enregistrements du rayonnement visible incident. Nous observons à partir du 3 mai une réduction de l'amplitude maximale due à une plus grande nébulosité observable par ailleurs sur les photographies effectuées
Figure 8. Diagramme de dispersion des mesures IT et $\mathrm{G}$ en fonction des mesures FR pour a) le rayonnement visible incident $\left(S W_{D N}\right)$;

b) le rayonnement visible réfléchi $\left(S W_{U P}\right)$;

c) le rayonnement infrarouge incident $\left(\mathrm{LW}_{\mathrm{DN}}\right)$;

d) le rayonnement infrarouge émis $\left(\mathrm{LW}_{\mathrm{UP}}\right)$.

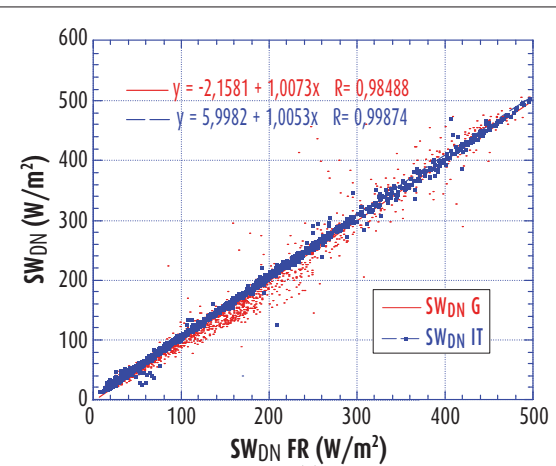

(a)

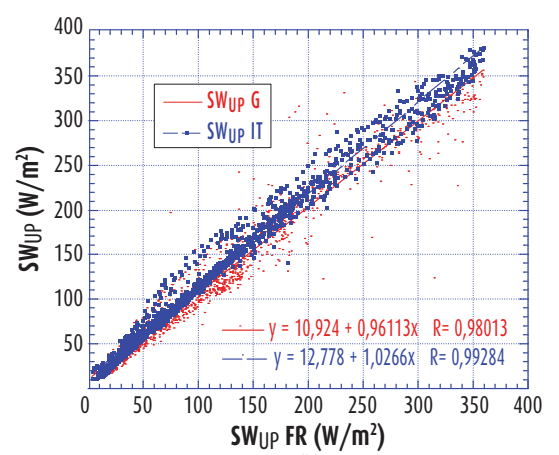

(b)

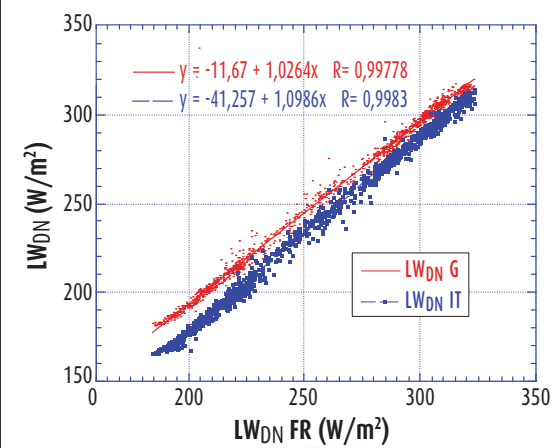

(c)

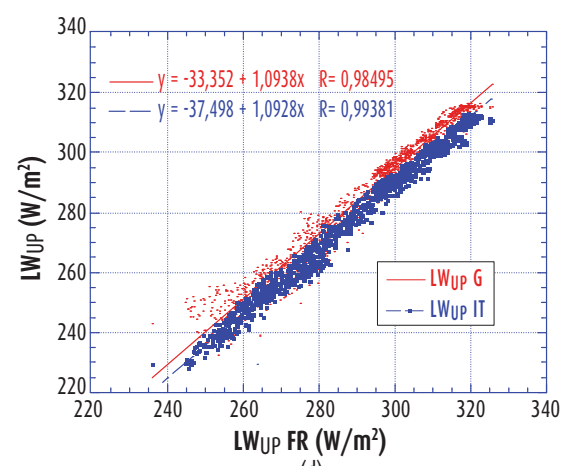

(d) 


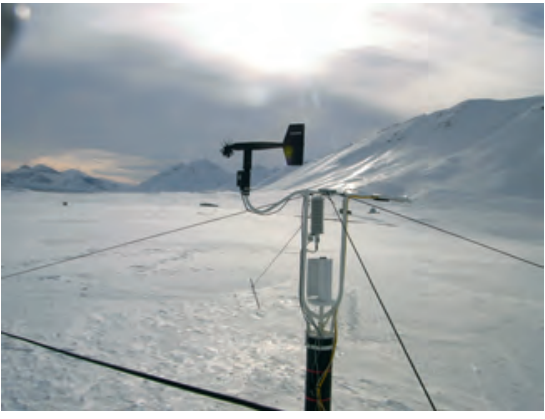

Photo 3 - Un exemple de la variabilité « spatiale » de la structure de la surface observée.

notera une dispersion importante dans ces corrélations, explicable, d'une part, par l'utilisation de capteurs et de niveaux de mesure différents et probablement, d'autre part, par des propriétés spécifiques variées des surfaces sous-jacentes, ce qu'illustre la photo 3 . On remarque en effet une structuration de la surface (différentes structures de neige et de glace) sous forme de facettes plus au moins gelées ou ayant subi une fonte au voisinage de neige plus lisse avec parfois des traces de passage.

Pour le rayonnement infrarouge, le rayonnement incident (fig. 8c) présente des niveaux de corrélation équivalents entre FR et IT, d'une part, et FR et G, d'autre part, même si le coefficient de corrélation est légèrement moindre avec G. Les pentes sont voisines de 1 mais le biais à l'origine est plus important entre FR et IT $\left(41 \mathrm{~W} / \mathrm{m}^{2}\right.$ contre $12 \mathrm{~W} / \mathrm{m}^{2}$ entre FR et $\mathrm{G}$ ). Bien que plus dispersées que pour le rayonnement infrarouge incident, les mesures de rayonnement infrarouge émis par la surface (fig. 8d) sont tout à fait comparables pour les trois stations, même si le coefficient de corrélation entre FR et $\mathrm{G}$ reste légèrement inférieur à celui entre FR et IT. Cependant les biais à l'origine sont forts $\left(37 \mathrm{~W} / \mathrm{m}^{2}\right.$ entre FR et IT, $33 \mathrm{~W} / \mathrm{m}^{2}$ entre FR et $\mathrm{G})$.

\section{Bilan d'énergie et incertitudes associées}

\section{Le rayonnement net}

Nous présentons tout d'abord les estimations du rayonnement net $(R n)$ et les comparaisons entre la station FR et les stations IT et G. Ces estimations sont importantes puisqu'elles viendront compléter les estimations des flux turbulents de chaleur sensible $(H)$ et latente $(E)$ afin d'accéder au flux de chaleur transmis à la glace $\operatorname{Tr}=R n-(H+E)$.

On retrouve pour les flux nets (figure 9) un cycle diurne prononcé jusqu'au 4 mai avec une alternance de valeurs fortement négatives (jusqu'à $-100 \mathrm{~W} / \mathrm{m}^{2}$ ) suivies de valeurs positives (jusqu'à $+90 \mathrm{~W} / \mathrm{m}^{2}$ ). À partir du 5 mai, l'amplitude du cycle diurne est significativement réduite en raison de valeurs nocturnes faiblement négatives voir proches de zéro. La période du 5 au 8 mai étant caractérisée par des flux incidents de courtes longueurs d'onde $\left(\mathrm{SW}_{\mathrm{DN}}\right)$ réduits (cf fig. 7), nous attribuons cette évolution du flux net à l'influence des nuages.

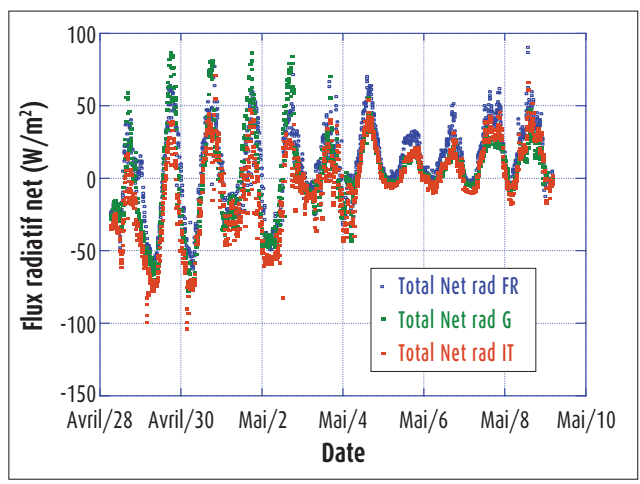

Figure 9 - Flux radiatifs nets FR, IT et G.

La figure 10 indique une dispersion importante des valeurs de flux net. Les valeurs estimées à partir du système BEAR sont toutefois cohérentes avec celles de la station IT (dans une moindre mesure avec celles de la station $\mathrm{G})$, avec une pente voisine de $1(0,89)$ et une corrélation élevée, de l'ordre de $0,93(0,83)$, malgré un biais à l'origine supérieur à $13 \mathrm{~W} / \mathrm{m}^{2}\left(5 \mathrm{~W} / \mathrm{m}^{2}\right)$. Cette forte dispersion du flux net ainsi que le biais observé entre les estimations FR et IT devront faire l'objet d'une analyse approfondie afin de ne pas

Figure 10 - Diagramme de dispersion des mesures IT et $G$ en fonction des mesures $F R$ pour le rayonnement net.

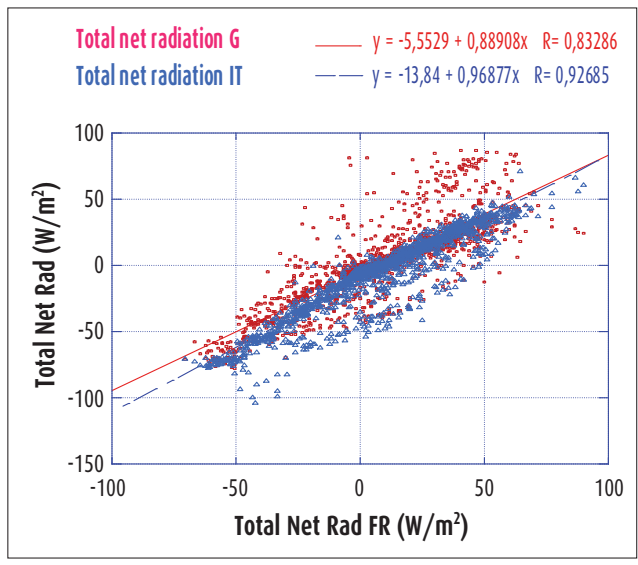

fausser, en cas de valeurs de flux net erronées, l'estimation du flux transmis à l'océan $T r$. Cela souligne tout l'intérêt du système ICE-T qui permettra d'accéder directement aux valeurs de $T r$ à partir de mesures dans la neige ou la glace.

\section{L’albédo}

L'albédo reste un paramètre difficile à estimer lorsque l'élévation solaire est faible. Au-dessous de 15 degrés, des variations fortes de l'albédo au-dessus de la neige ont déjà été constatées et reliées à plusieurs facteurs tels que la mauvaise réponse en cosinus du pyranomètre, la variation de la composition spectrale solaire à élévation faible (Kondratyev, 1954) et la réflexion spéculaire à la surface de la neige. Différents travaux (McGuffie et Henderson-Sellers, 1985 ; Dubreuil et Woo, 1984) ont en particulier montré la nécessité d'évaluer l'albédo au maximum solaire afin de limiter les artefacts radiatifs difficilement corrigeables, dus notamment à la topographie de la surface de neige et aux relations avec l'élévation.

Comme l'élévation solaire est encore basse dans cette région à cette période de l'année (moins de 17 degrés à $6 \mathrm{~h}$ UTC et autour de 25 degrés entre $9 \mathrm{~h}$ et $13 \mathrm{~h}$ UTC), nous nous intéresserons essentiellement aux mesures correspondant aux élévations solaires les plus élevées afin d'éviter de prendre en compte des mesures erronées. Nous avons donc choisi de ne présenter les mesures d'albédo qu'au voisinage du maximum solaire, observé vers 11 h UTC.

Sur la figure 11, les mesures d'albédo entre $10 \mathrm{~h} 30$ et $13 \mathrm{~h} 00$ UTC sont assez dispersées bien que présentant un plateau entre 0,7 et 0,9 . La figure 11 témoigne ainsi de la difficulté de la mesure, particularité déjà remarquée par McGuffie et Henderson-Sellers (1985) lors de mesures au-dessus de la neige. Sachant que l'élévation solaire à la période de mesure n'excède pas 26 degrés, les mesures d'albédo que nous avons effectuées sont en outre susceptibles de prendre en compte des effets potentiels de « sur- ou sous- » réflexion dus à la surface (Yamanouchi, 1983), d'autant que la topographie de la surface du champ de mesure présente des 


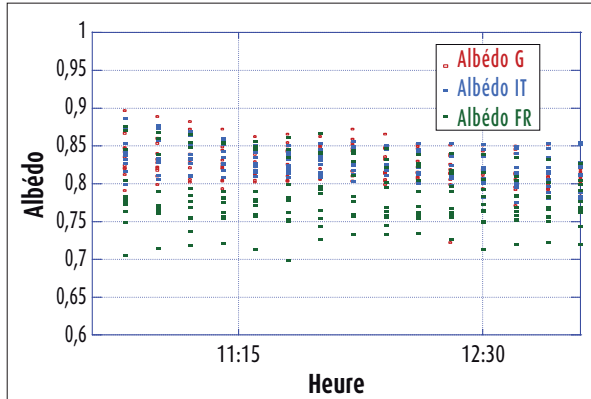

Figure 11 - Mesures de l'albédo sur les trois stations FR, IT et $G$ entre $10 \mathrm{~h} 30$ et $13 \mathrm{~h}$ UTC.

ruptures ou sastrugi $i^{(1)}$, comme le montre la photo 4 pour un petit élément de quelques mètres carrés.

Il est difficile de déduire des mesures une valeur d'albédo qui soit représentative de la surface pour chacune des stations FR, IT et G. Une valeur moyenne avec son écart-type est sans doute plus pertinente mais souligne une différence dans la nature des surfaces observées par les trois radiomètres :

- mesures FR : albédo $=0,773 \pm 0,029$;

- mesures IT : albédo $=0,833 \pm 0,024$;

- mesures $\mathrm{G}:$ albédo $=0,837 \pm 0,023$.

Photo 4 - Champ de vue sous BEAR montrant des hétérogénéités de topographie sur une surface de quelques mètres carrés.

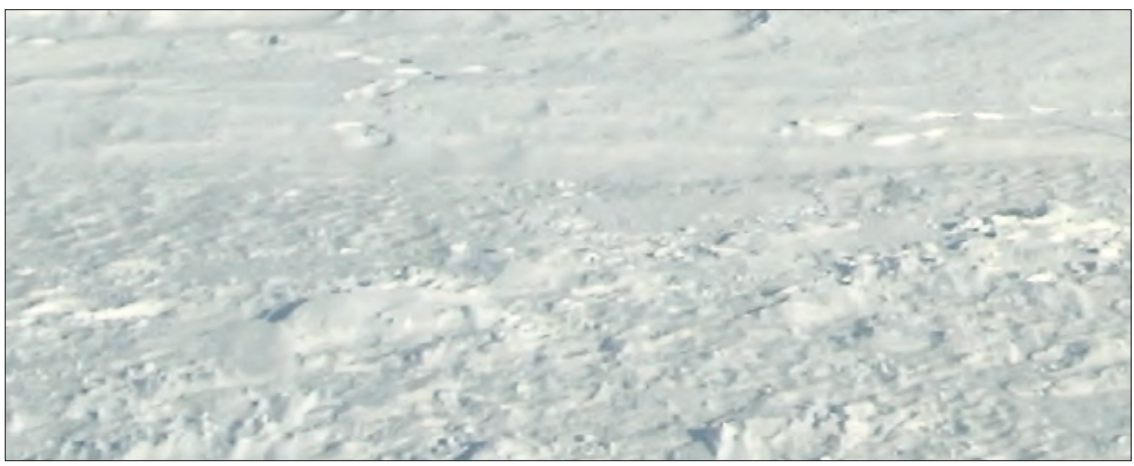

Nous avons donc une inquiétude quant à la représentativité de l'évaluation de l'albédo par BEAR qui serait liée à la nature de la surface vue par le CNR4 et qui justifierait des photographies systématiques pour avoir une idée des échelles d'hétérogénéité représentatives de la surface.

Par ailleurs, un biais (ou différence à l'origine) supérieur à $10 \mathrm{~W} / \mathrm{m}^{2}$ a été trouvé sur la composante visible réfléchie (fig. 8b) avec un niveau de corrélation élevé et une pente voisine de 1 , aussi bien entre FR et IT qu'entre FR et $\mathrm{G}$. Ce résultat va dégrader les corrélations d'albédo entre FR et IT et FR et G, surtout aux faibles valeurs du rayonnement visible réfléchi. Sans surprise, on trouve un biais de 0,39 , une pente de 0,6 et un coefficient de corrélation de seulement 0,68 entre les valeurs d'albédo FR et IT, et du même ordre entre FR et G. surface (sous le radiomètre de BEAR).
Cependant, si nous nous intéressons, par exemple, aux mesures d'albédo à l'heure du maximum solaire, celles-ci deviennent plus proches et paraissent en relation avec le type de surface observée (tableau 1). En particulier, le 29 avril, jour où il a neigé sur l'ensemble des sites, on trouve une valeur moyenne de l'albédo mesurée par le système BEAR de 0,85 , certes inférieure de $2,3 \%$ à celles obtenues à partir des mesures IT et $\mathrm{G}$ mais relativement proche de celles-ci ( 0,87 pour les deux stations). Ce n'est pas le cas pour les autres journées où l'albédo FR est nettement inférieur aux albédos G et IT.

\section{La température de la surface de la neige Ts}

Nous avons utilisé comme valeurs de référence la moyenne des mesures de température à $0,5 \mathrm{~cm}$ sous la surface de la neige effectuées par MétéoFrance CENG à partir de quatre sondes de température PT100. Ce jeu de données de référence (notées $T_{C E N G}$ ) nous a ainsi permis d'évaluer les températures de surface $T s$ obtenues par inversion du rayonnement infrarouge : $L W_{U P}-(1-\varepsilon) L W_{D N}=\varepsilon . \sigma . T_{s}^{4}$ (Claffey et al., 1999) et mesurées par les trois stations FR, IT et G.

Dans un premier temps, nous avons comparé les valeurs de température de surface de la neige $T s$ obtenues par l'équipe italienne par inversion du rayonnement infrarouge $L W_{U P}$ mesuré au sommet de la tour pour différentes

\begin{tabular}{|lllll|}
\hline Date & $\mathbf{2 8}$ avril & $\mathbf{2 9}$ avril & $\mathbf{2}$ mai & $\mathbf{6}$ mai \\
\hline Surface observée sur le site FR & $\begin{array}{l}\text { Neige } \\
\text { glacée }\end{array}$ & $\begin{array}{l}\text { Neige } \\
\text { fraîche }\end{array}$ & $\begin{array}{l}\text { Neige } \\
\text { glacée }\end{array}$ & $\begin{array}{l}\text { Neige légère } \\
\text { soufflée }\end{array}$ \\
\hline Albédo FR & 0,75 & 0,85 & 0,78 & 0,79 \\
\hline Albédo G & 0,82 & 0,87 & 0,83 & 0,85 \\
\hline Albédo IT & 0,83 & 0,87 & 0,83 & 0,85 \\
\hline Tableau 1- Valeur moyenne de l'albédo mesuré au voisinage du maximum solaire pour différents états de la
\end{tabular}

émissivités. Les niveaux de corrélation entre $T_{s}(\mathrm{IT})$ et $T_{C E N G}$ sont élevés pour toutes les émissivités choisies entre 0,95 et 0,99 . Toutefois, la valeur de 0,99 minimise le biais à l'origine $\left(0,58{ }^{\circ} \mathrm{C}\right)$ pour une pente de régression voisine de 1 .

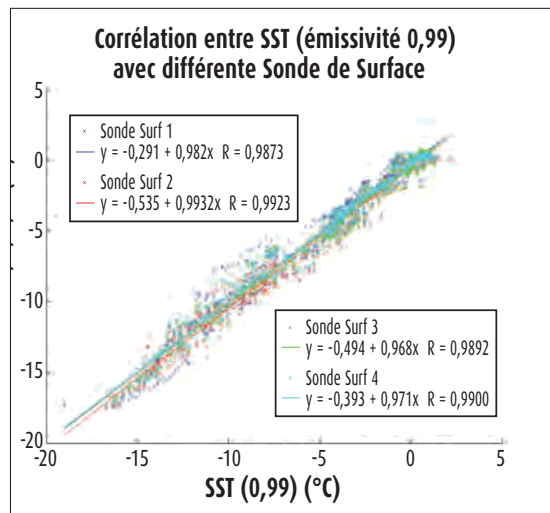

Figure 12 - Diagramme de dispersion de la tempé rature à $0,5 \mathrm{~cm}$ sous la surface mesurée par les quatre sondes du CENG en fonction de la température de la surface de neige BEAR ( $T_{s}$ inversée pour une émissivité de 0,99).

Une émissivité de 0,99 s'avère donc optimale pour la campagne de mesure, ce qui est conforme aux résultats de Claffey et al. (1999) et Persson et al. (2002). Par la suite, nous avons donc choisi de garder cette valeur pour le calcul de la température de surface de la neige pour l'ensemble des stations.

La figure 12 montre que les valeurs de température de surface ainsi estimées pour la station BEAR - notées $T s(\mathrm{FR})$ sont très corrélées avec les valeurs de température à $0,5 \mathrm{~cm}$ sous la surface de la neige mesurées par les quatre sondes, avec un biais à l'origine en général inférieur à $-0,53{ }^{\circ} \mathrm{C}$. La régression entre Ts $(\mathrm{FR})$ et la température moyenne

(1) Un sastruga (mot emprunté au russe, généra lement utilisé au pluriel sastrugi) est une irrégularité topographique tranchante sur une surface de neige résultant de l'érosion causée par le vent, de la saltation (déplacement par sauts) de particules de neige et de leur déposition dans les régions enneigées, polaires comme tempérées. 
$T_{\text {CENG }}$ donne un coefficient de corrélation de 0,99 , une pente de 0,979 et un biais à l'origine de $-0,42{ }^{\circ} \mathrm{C}$.

Par ailleurs, les corrélations entre $T s(\mathrm{FR})$ et $T s(\mathrm{IT})$, d'une part, et $T s(\mathrm{FR})$ et $T s(\mathrm{G})$, d'autre part, sont satisfaisantes : les pentes sont voisines de 1 mais les différences à l'origine sont élevées. Cela aura des conséquences sur l'évaluation des flux turbulents bulk.

\section{Évaluation des flux de chaleur sensible et latente bulk - Incertitudes associées}

Nous nous trouvons dans des conditions de transition de printemps pendant lesquelles les couches de surface sont très ténues. Dans ce contexte, l'approche bulk de Price et Dunne (1976) et Arck et Sherer (2002) est appropriée et a été appliquée en choisissant une longueur de rugosité aérodynamique égale à $10^{-4} \mathrm{~m}$. Les figures 13 et 14 montrent les comparaisons des résultats de cette même méthode appliquée aux différents instruments et sites FR, G et IT.

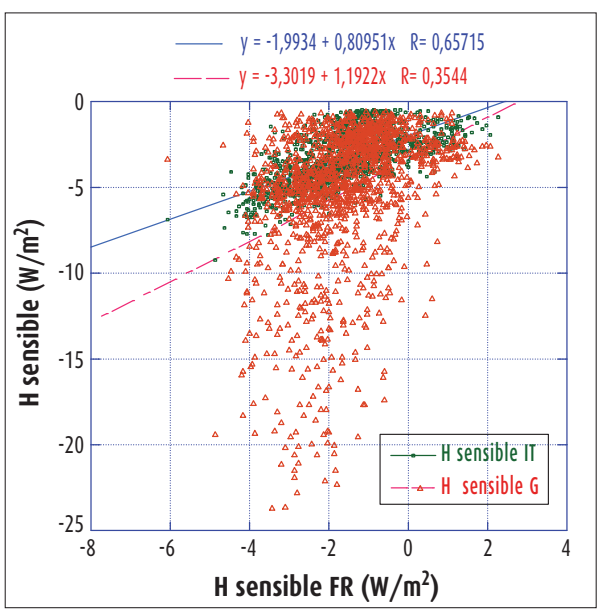

Figure 13 - Diagramme de dispersion des estimations IT et $\mathrm{G}$ en fonction des estimations FR pour le flux de chaleur sensible bulk.

On constate que les estimations des flux de chaleur sensible FR et IT sont proches, même si la dispersion est importante (fig. 13). Les mesures de G paraissent très différentes en raison de valeurs très négatives dues à de forts écarts entre la température de surface de la neige $T s$ et la température de l'air mais, en réalité, la majeure partie des flux $\mathrm{G}$ a des valeurs proches et corrélées avec celles de FR et IT.

En ce qui concerne le flux de chaleur latente (fig. 14), on remarque que FR et IT ont un comportement identique. Le

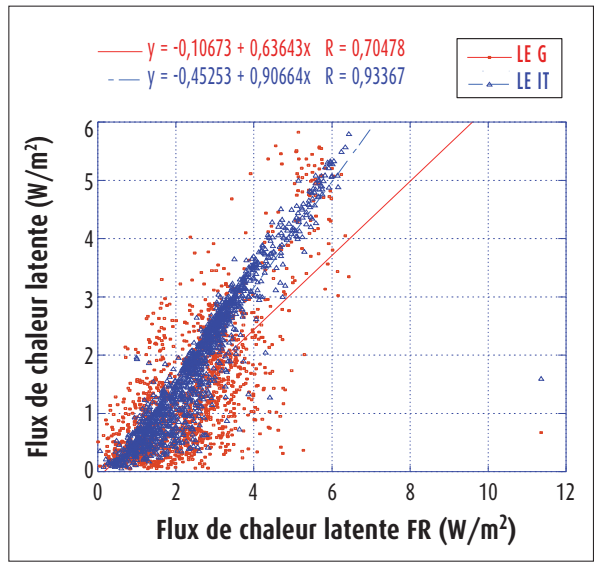

$10 \mathrm{~cm}$ ) par rapport à 1,7 m entraînerait un biais sur les flux estimés de $0,6 \%$ (respectivement $1,2 \%$ ); - une variation de $h$ de $50 \%$ entrầnerait un biais sur les flux estimés de $10 \%$ pour la même longueur de rugosité, et de $39 \%$ si $\mathrm{z}_{0}=10^{-2} \mathrm{~m}$.

Il y a donc lieu de faire très attention à la mesure de la hauteur de la neige.

Analysons maintenant l'incertitude liée à une méconnaissance de la longueur de rugosité. Nous supposons dans ce cas que $h$ est connu et nous prenons $h=1,7 \mathrm{~m}$ correspondant à nos observations. Pour avoir une

Figure 14 - Diagramme de dispersion des estimations IT et $\mathrm{G}$ en fonction des estimations FR pour le flux de chaleur latente bulk.

biais à l'origine est petit, ce qui est cohérent avec les résultats obtenus pour les variables météorologiques (nous avions déjà vu que les humidités spécifiques et les intensités du vent aux deux stations étaient très proches). En revanche, la comparaison entre FR et G témoigne d'un comportement différent sur des sites de caractéristiques locales différentes. En effet, nous avions remarqué que l'intensité du vent, la température de l'air et l'humidité relative y étaient différentes.

Notre objectif est maintenant de délimiter les plages d'incertitude susceptibles d'affecter notre estimation des flux turbulents. Il nous faut tout d'abord estimer les incertitudes sur les flux que peuvent entraîner une mesure erronée de la hauteur $h$ des capteurs par rapport à la surface (par exemple dans le cas de nonconnaissance de la distance des capteurs au plan zéro suite à des variations de la hauteur de neige) mais également l'incertitude sur la longueur de rugosité de la surface $\mathrm{z}_{0}$. En effet, ces deux grandeurs interviennent dans les formulations bulk utilisées. D'autre part, l'incertitude liée à la (non) prise en compte d'une longueur de rugosité spécifique pour la température et l'humidité doit également être quantifiée.

En ce qui concerne l'incertitude liée à une méconnaissance de la hauteur $h$ des capteurs par rapport à la surface de neige/glace, nous avons tout d'abord vérifié que la hauteur de la neige n'avait pas varié de plus de quelques centimètres au cours de l'expérience. Des tests de sensibilité au paramètre $h$ nous indiquent que :

- pour une longueur de rugosité aérodynamique $\mathrm{z}_{0}$ choisie égale à $10^{-4} \mathrm{~m}$, une variation de $h$ de $5 \mathrm{~cm}$ (respectivement incertitude de moins de $10 \%$ sur les flux, il faudrait que l'incertitude sur $\mathrm{z}_{0}$ (notée $\mathrm{dz}_{0}$ ) soit inférieure à 4,9 $10^{-4} \mathrm{~m}$ pour $\mathrm{z}_{0}=10^{-3} \mathrm{~m}$, et que $\mathrm{dz}_{0}$ soit inférieure à $2,610^{-3} \mathrm{~m}$ pour $\mathrm{z}_{0}=10^{-2} \mathrm{~m}$. Ce domaine d'incertitude sur $\mathrm{z}_{0}$ n'est pas trop contraignant mais il signifie que la gamme des longueurs de rugosité qui peuvent survenir aura une incidence importante sur les flux.

En effet, nous n'avons pas accès à une estimation précise de la longueur de rugosité mais plutôt à une valeur « moyenne » liée à une typologie de la surface de neige. On conçoit donc l'importance de ce paramètre et l'utilité d'observations de la texture de la surface par photographies systématiques. En particulier, les mesures d'albédo peuvent contribuer à lever le doute sur les longueurs de rugosité, non pas en termes de connaissance précise de leurs valeurs mais plutôt pour discriminer leur ordre de grandeur et encadrer les précisions des mesures.

C'est là que la proposition d'Andreas effets classiques liés à la viscosité et à la turbulence selon Charnock (1955), cette paramétrisation prend en compte un effet de structuration spatiale macroscopique de la surface de neige.

Nous avons repris les estimations des flux en utilisant cette paramétrisation d'Andreas et al. (2005). La modification moyenne associée à cette paramétrisation est une augmentation des flux de $30 \%$, que ce soit pour le flux de chaleur latente ou pour le flux de chaleur sensible.

Enfin, si nous prenons en compte les longueurs de rugosité thermique en calculant les nombres de Reynolds turbulents de surface $\left(R_{t}=z_{0} U_{*} / v\right)$ selon Andreas et al. (2005), les flux de chaleur sensible et latente sont réduits et al. (2005) intervient car, en plus des 
en moyenne de $10 \%$ par rapport aux estimations obtenues en utilisant la longueur de rugosité aérodynamique, avec un écart-type voisin de $30 \%$.

\section{Bilan d'énergie en régions arctiques}

Pour l'expérience que nous venons de mener, cette gamme d'incertitude sur les flux turbulents de chaleur n'a que peu d'incidence sur le bilan d'énergie. Pour nous en convaincre, nous avons représenté sur la même figure (figure 15) le rayonnement net total $\left(R_{n}\right.$, en bleu) et l'énergie transmise $\left(T_{r}=R_{n}-(H+E)\right.$, en rouge). Dans la période considérée, le rayonnement net a beaucoup plus d'importance pour la surface que les flux turbulents de chaleur, d'autant que le flux net infrarouge est nettement supérieur en valeur absolue aux flux turbulents de chaleur, y compris pendant la nuit.

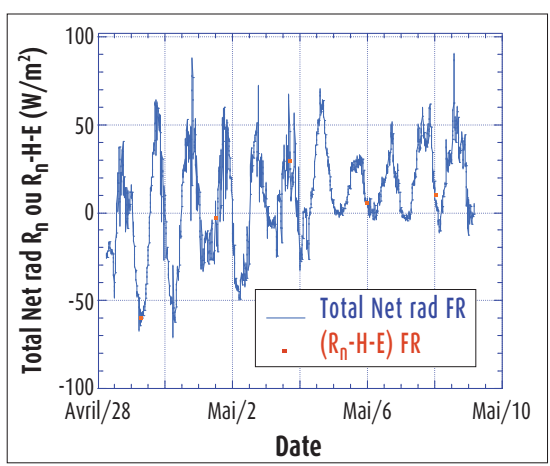

Figure 15 - Rayonnement net total $\left(R_{n}\right.$, en bleu) et énergie transmise $\left[T_{r}=R_{n}-(H+E)\right]$, en rouge, en fonction du temps pour la station BEAR.

Afin d'anticiper l'utilisation future de la station BEAR dans la région du Spitzberg dans le cadre du projet OPTIMISM, il convient de remarquer (Persson et al., 2002) que la somme des flux turbulents de chaleur $(H+E)$ est petite et négative en moyenne mais contribue au bilan radiatif pour environ $20 \%$, en chauffant la surface pendant l'hiver et au mois de juillet et en la refroidissant en mai, juin et août. En admettant une incertitude de $50 \%$ sur les valeurs des flux turbulents horaires de chaleur, aura-t-on une chance d'estimer correctement leurs contributions au bilan? En supposant que les biais soient petits et en prenant des échantillons horaires indépendants, l'incertitude devrait être réduite à $4,2 \%$ sur les flux journaliers, ce qui devrait nous permettre de bien analyser le bilan d'énergie à la surface tout au long de l'année. Cette hypothèse de quasi-stationnarité des flux n'a de sens que si et seulement si la variabilité temporelle des flux est faible (cela ne s'applique donc pas entre avril et août). Toutefois, la prise en compte du cycle diurne, s'il est dominant, ne devrait pas trop dégrader cette précision.

\section{Conclusions}

Dans un premier temps, nous avons vu qu'il convient de rester vigilant sur la question du givrage bien que les instruments choisis aient généralement été utilisés en montagne, en Arctique et en Antarctique. Un critère de risque de givrage par températures faiblement négatives et forte humidité par rapport à la glace pourrait être évalué en l'absence d'un critère optimisé prenant en compte d'autres paramètres tels que le vent (voir le rapport COST 727, 2006 sur le givrage). Des séquences de mesures avec chauffage et sans chauffage, choisies de telle sorte qu'elles soient représentatives de l'évolution de l'atmosphère et de la surface sur une année, devront être effectuées afin d'évaluer l'énergie nécessaire pour chauffer les capteurs radiatifs. Par ailleurs, les capteurs radiatifs devront être étalonnés avant et après l'expérience pour lever le doute sur des biais instrumentaux.

En ce qui concerne la qualité des mesures effectuées par le système BEAR, nous avons obtenu les résultats suivants :

- pour les variables météorologiques, le système BEAR fournit des données très proches des mesures italiennes sur le même site à Ny-Ålesund ;

- pour les mesures radiatives, les composantes considérées une à une sont très proches, surtout les rayonnements incidents pour lesquels les biais sont petits. En revanche, les composantes réfléchies mesurées par BEAR présentent des biais (ou écarts) dus aux effets de surface qui se répercutent surtout sur les mesures de l'albédo et de façon moindre sur le rayonnement net ;

- pour la température de surface $T_{s}$, une émissivité de 0,99 a été trouvée optimale, en accord avec les considérations de Claffey et al. (1999). Cette valeur en effet :

a) minimise les écarts avec les mesures à la surface de la neige effectuées par Météo-France et permet d'obtenir une pente voisine de 1 ainsi que des coefficients de corrélation supérieurs ou égaux à 0,9 ; b) minimise les biais par rapport aux valeurs italiennes, sachant toutefois que ces valeurs sont estimées par inversion du rayonnement infrarouge émis par la surface et mesuré au sommet du mât à $32 \mathrm{~m}$ et qu'elles ne sont pas corrigées, ni en température ni en humidité.

Des mesures de Ts pour des conditions de surface variées de neige ou de glace, ou la combinaison des deux, nous permettront d'analyser si ce choix d'émissivité reste pertinent.

- Les mesures d'albédo sont délicates et doivent être effectuées avec précaution en centrant la période de mesure autour du maximum d'élévation solaire afin de ne pas être affectées par des effets parasites liés à la surface. Dans la mesure où elles sont très sensibles, par exemple, à la topographie et aux phénomènes de réflexion spéculaire, il conviendrait de les accompagner de photographies de la surface, ce qui permettrait de statuer sur la qualité des mesures et de discriminer celles-ci en fonction des longueurs de rugosité estimées à partir des photographies. Il paraît également intéressant de comparer les mesures d'albédo (en ciel clair) à des mesures spatiales qui présentent d'autres difficultés d'interprétation (Curry et al., 2000).

- Les flux que nous avons estimés ont été déterminés par une formulation bulk qui peut s'appliquer aux transitions de printemps (Arck et Sherer, 2002). Les différences que nous avons trouvées avec les mesures du mât italien semblent provenir essentiellement de la température de la surface. Ceci justifie de ne pas se contenter de la seule inversion des mesures radiatives fournies par la station BEAR mais d'utiliser aussi les mesures de température de la surface qui seront fournies par le système ICE-T, ce qui correspond au choix annoncé dans le projet OPTIMISM.

Nous avons pu montrer que les incertitudes sur la distance à la surface (plan zéro) et sur les longueurs de rugosité de la surface pouvaient avoir une importance considérable pour l'estimation des flux turbulents de chaleur. Une étape supplémentaire consistera à prendre en compte les relations les plus plausibles entre longueurs de rugosité aérodynamiques et thermiques ou hydriques. Enfin, pour bien encadrer les flux et le bilan d'énergie, les plages d'incertitudes associées à différentes paramétrisations (en plus de celle de Arck et Sherer, 2002) restent à déterminer. 
Dans le cadre du développement et des premières utilisations en conditions réelles du système BEAR, nous avons eu la chance de pouvoir comparer les mesures produites par ce système avec d'autres mesures issues de différentes plateformes, ce qui nous a conforté dans nos choix instrumentaux et dans la logique du projet. Le fonctionnement en vraie grandeur $\mathrm{du}$ système OPTIMISM devrait encore susciter un certain nombre de questions d'ordres technique et scientifique, dont le givrage qui n'en est pas une des moindres.

\section{Remerciements}

Le projet OPTIMISM est soutenu par l'Agence nationale de la recherche (ANR-09 BLANC 02277602) et l'Institut Paul-Émile Victor (IPEV, programme 1015) et il a reçu un soutien incitatif de LEFE (Les enveloppes fluides et l'environnement) - INSU/ CNRS.

Un très grand remerciement aux équipes italiennes d'ISAC (CNR) et d'AWI (Allemagne) pour la mise à disposition de leurs données relatives à l'expérience d'avril-mai 2010 à Ny-Ålesund.

Nous remercions particulièrement les rapporteurs de l'article pour l'analyse approfondie du texte, leurs suggestions pertinentes et les corrections suggérées.

\section{Bibliographie}

\footnotetext{
- Andreas E. L., 2002 : Parameterizing scalar transfer over snow and sea ice: a review. J. Hydrol., 3, 417-432.

- Andreas E. L., E. R. Jordan et A. P. Makshtas, 2005 : Parameterizing turbulent exchanges over sea ice: the Ice Station Weddell results. Bound.-Layer Meteor., 114, 439-460.

- Arck M. et D. Sherer, 2002 : Problems in the determination of sensible heat flux over snow. Geografiska Annaler, 84A, 3-4.

- Beljaars A. C. M et A. A. M. Holtslag, 1991 : Flux parameterization over land surfaces for atmospheric models. J. Appl. Meteor., $30,327-341$.

- Brunke M. A., M. Zhou, X. Zeng et E. L Andreas, 2006 : An intercomparison of bulk aerodynamic algorithms used over sea ice with data from the SHEBA experiment. - J. Geophys. Res., 111, C09001, D0l:10.1029/2005JC002907.

- Businger J. A., J. C. Wyngaard, Y. Izumi et E. F. Bradley, 1971 : Flux-profile relationships in the atmospheric surface layer. J. Atmos. Sci., 28, $181-189$.

- Charnock H., 1955 : Wind stress on a water surface. Quart. J. Roy. Meteor. Soc., 81, 639-640.

- Claffey K. A., E. L. Andreas, D. K. Perovich, C. W. Fairall, P. S. Guest et P. O. G. Persson, 1999 : Surface temperature measurement at SHEBA. In preprints Fifth - Conference on Polar Meteorology and Oceanography, 10-15 janvier 1999, Dallas, Texas, 86-90, Amer. Meteor. Soc.

- COST 727, 2006 : Atmospheric icing on structures measurements and data collection on icing: state of art. ESF, publication de MeteoSwiss, 75, $110 \mathrm{p}$.

Curry J. A., P. V. Hobbs, M. D. King, D. A. Randall, P. Minnis, G. A. Isaac, J. O. Pinto, T. Uttal, A. Bucholtz, D. G. Cripe, H. Gerber, C. W. Fairall, T. J. Garrett, J. Hudson, J. M. Intrieri, C. Jakob, T. Jensen, P. Lawson, D. Marcotte, L. Nguyen, P. Pilewskie, A. Rangno, D. C. Rogers, K. B. Strawbridge, F. P. J. Valero, A. G. Williams et D. Wylie, 2000 : FIRE Arctic Clouds Experiment. Bull. Amer. Meteor. Soc., 81, 5-30.

- De Bruin H. A. R., R. J. Ronda et B. J. H. Van De Wiel, 2000 : Approximate solutions for the Obukhov length and the surface fluxes in terms of bulk Richardson number. Bound.-Layer Meteor., 95, 145-157.

- Dubreuil M. A. et M. K. Woo, 1984 : Problems of determining snow albedo for the high arctic. Atmos. Ocean, 22, 3, 379-386

- Fairall C. W., E. F. Bradley, J. E. Hare, A. A. Grachev et J. B. Edson, 2003 : Bulk parameterization of air-sea fluxes: updates and verification for the COARE Algorithm. J. Climate, 16, 571-591.

- Grachev A. A. et C. W. Fairall, 1997 : Dependence of the Monin-Obukhov stability parameter on the bulk Richardson number over the ocean. J. Appl. Meteor., 36, 406-414.

Grachev A. A., E. L Andreas, C. W. Fairall, P. S. Guest et P. O. G. Persson, 2007 : SHEBA flux-profile relationships in the stable atmospheric boundary layer. Bound.- Layer Meteor., 124, 315-333.

- Kondratyev K. Y. A., 1954 : Albedo of underlying surface and cloud. The radiant energy of the sun. Gidromet Leningrad (sommaire anglais preparé par A. Kurdents et P. Larson, McGill University, Montréal).

- Lüers J. et J. Bareiss, 2010 : The effect of misleading surface temperature estimations on the sensible heat fluxes at a high arctic site - the Artic Turbulence Experiment 2006 on Svalbard (ARCTEX-2006). Atmos. Chem. Phys., 10, 157-158.

McGuffie K. et A. Henderson-Sellers, 1985 : The diurnal hysteresis of snow albedo. J. Glaciol., 31, 108, 188-189.

- Perovich D. K., T. C. Grenfell, B. Light et P. V. Hobbs, 2002 : Seasonnal evolution of the albedo of multiyear arctic sea ice. J. Geophys. Res., 107, C10, 8044, DOI:10.1029/2000JC000438.

- Persson P. O. G, C. W. Fairall, E. L. Andreas, P. S. Guest et D. K. Perovich, 2002 : Measurement near the atmospheric surface flux tower at SHEBA: near-surface - conditions and surface energy budget. J. Geophys. Res., 107, C10, 8045, D0I:10.1029/2000JC000705.

- Price A. G. et T. Dunne, 1976 : Energy balance computations of snowmelt in a subarctic area. Water Res., 12, 4, 686-694.

- UNAVCO, 2008 : Summary report on Forgen 500 wind turbine performance, 5 p.

Vivier F., 2010 : http://optimism.locean-ipsl.upmc.fr/tiki-index.php.

- Weill A., 1991 : Indirect measurement of fluxes using Doppler sodar. In Land Surface Evaporation, Springer-Verlag, T. Schmugge et J. S. André éditeurs, $301-311$.

- Yamanouchi T., 1983 : Variations of incident solar flux and snow albedo on the solar zenith angle and cloud cover at Mizuho station, Antarctica. J. Meteor. Soc. Japan, 61, 879-893
} 